\title{
Rates of Transmitting Erwinia stewartii from Seed to Seedlings of a Sweet Corn Hybrid Susceptible to Stewart's Wilt
}

\author{
P. M. Michener, J. K. Pataky, and D. G. White, Department of Crop Sciences, University of Illinois, Urbana \\ 61801
}

\begin{abstract}
Michener, P. M., Pataky, J. K., and White, D. G. 2002. Rates of transmitting Erwinia stewartii from seed to seedlings of a sweet corn hybrid susceptible to Stewart's wilt. Plant Dis. 86:10311035 .

Rates of transmitting Erwinia stewartii from seed to seedlings were estimated from field growouts of seedlings grown from seed infected with E. stewartii. Infected seed were produced in 1998, 1999, and 2000 on a Stewart's wilt-susceptible sweet corn hybrid, Jubilee. Seedlings were inoculated repeatedly with pinprick inoculators and suspensions of $E$. stewartii were injected into ear shanks of the primary ears of each adult plant. Seed from inoculated plants were harvested and bulked. Single kernels were assayed for E. stewartii to estimate the proportion of kernels infected with $E$. stewartii. Estimates of $E$. stewartii-infection were $15.6 \pm 4.3,49.4 \pm$ 3.9 , and $12.5 \pm 2.4 \%$ for seed produced in 1998, 1999, and 2000, respectively. Approximately 61,800 seedlings were grown in DeKalb, IL in 1999 and 83,400 and 60,000 seedlings were grown in Plover WI in 2000 and 2001, respectively, from infected seed lots produced the previous year. Approximately 10,000, 12,200, and 29,400 seedlings of susceptible sweet corn hybrids also were grown each year from commercial seed produced in Idaho where Stewart's wilt does not occur. Based on estimates of kernel infection in each seed lot and plant populations in each grow-out trial, about 9,600, 41,200, and 7,500 seedlings were grown from infected kernels in 1999, 2000, and 2001, respectively. Seedlings at the two- to three-leaf stage were examined for symptoms of Stewart's wilt. Infected plants were confirmed by microscopic observations of bacterial ooze and by enzyme-linked immunosorbent assay. When data were combined from all three trials, 59 of approximately 58,300 seedlings grown from infected seed were infected with E. stewartii based on symptoms of Stewart's wilt and E. stewartii-positive leaf tissue samples. Of these 59 seedlings, 22 probably were infected from seed-to-seedling transmission of $E$. stewartii and 37 probably were the result of natural infection due to the presence of flea beetles in DeKalb in 1999. Twenty-two infected seedlings from 58,300 infected kernels corresponds to a seed-to-seedling transmission rate of $0.038 \%$. This rate of seed-to-seedling transmission of $E$. stewartii is substantially lower than seed transmission rates reported in the first half of the twentieth century; however, it is similar to seed-to-seedling transmission rates reported from other recent research.
\end{abstract}

Stewart's bacterial wilt, caused by Erwinia stewartii (syn. Pantoea stewartii), occurs primarily in the mid-Atlantic states, the Ohio River Valley, and the southern part of the United States corn belt. The corn flea beetle, Chaetocnema pulicaria Melsh., is the vector and overwintering host of E. stewartii $(8,9)$. Other insects, primarily the toothed flea beetle Chaetocnema denticulate, were transient vectors of E. stewartii in controlled greenhouse trials, but none have been documented as significant vectors of $E$. stewartii in field situations (9). Winter survival of flea

Corresponding author: J. K. Pataky

E-mail: j-pataky@uiuc.edu

Partial funding provided by the Illinois Council on Food and Agricultural Research (CFAR) Grant No. 00I-12-3-CS.

Accepted for publication 30 April 2002.

Publication no. D-2002-0722-01R

(C) 2002 The American Phytopathological Society beetles is affected by temperature (20). Based on daily temperatures in the United States corn belt, the winters of 1991-92, 1997-98, 1998-99, and 1999-2000 were among the 10 warmest in the past 100 years. Consequently, flea beetles survived farther north in the United States, and Stewart's wilt occurred in many areas of the United States corn belt where seed was produced in 1992, 1998, 1999, and 2000.

Many countries prohibit or place restrictions on importation of maize seed produced in the United States to prevent the introduction of E. stewartii. When maize seed is produced in fields from which Stewart's wilt is identified, seed must be certified free of E. stewartii based on a greenhouse grow-out test or other procedures. An efficient seed health test based on an enzyme-linked immunosorbent assay (ELISA) can be used to detect seedborne E. stewartii (12). Polymerase chain reactions (PCRs) that detect E. stewartii have the potential to be adapted for seed health tests $(1,2,6)$.
Block et al. (3) maintained that the risk of introducing E. stewartii on good quality seed produced on a moderately resistant seed parent has been exaggerated greatly. Reports of seed transmission of E. stewartii that appeared in the literature from about 1900 to 1930 were based on trials in which a relatively few seed of highly susceptible germ plasm were evaluated or dissemination of E. stewartii by corn flea beetles was overlooked $(8,10,16,17,19,21)$. For example, Smith (19) reported 9\% seedling infection in 1909 and Thomas (21) observed transmission of E. stewartii to $85 \%$ of plants grown in a greenhouse in 1924. Neither of these reports considered flea beetle vectors or fungal pathogens that produce wilt symptoms. In 1933, Rand and Cash (17) assayed about 2 liters of "the worst-looking seed" from a highly susceptible cultivar and reported that $2 \%$ of seedlings grown from this seed in a greenhouse had symptoms of Stewart's wilt. Subsequently, a $2 \%$ rate of seed transmission for E. stewartii was reported in authoritative monographs on Stewart's wilt $(7,15,18)$. In the past decade, research on seed transmission of E. stewartii produced transmission rates that were considerably lower than those reported in previous literature $(3,4,11)$, which suggests that the estimate of $2 \%$ seed transmission does not accurately reflect the risk of introducing $E$. stewartii in seed.

Seed transmission of E. stewartii involves two processes, as illustrated by the schematic diagram in a recent review of the epidemiology of seedborne diseases (13). First, kernels are infected on seedparent plants in seed production fields (i.e., plant-to-seed transmission of E. stewartii). When infected seed are planted, plants can become infected if E. stewartii is transmitted from infected kernels to seedlings (i.e., seed-to-seedling transmission). The risk of introducing $E$. stewartii on seed to areas where it does not occur is affected by both of these processes and factors that influence them.

An ELISA-based seed health test developed in the past decade accurately detects seedborne E. stewartii (12). This test can be used to estimate relatively low levels of E. stewartii-infected kernels $(4,12,14)$. If the proportion of infected seed is estimated accurately from seed health tests, the risk of introducing E. stewartii can be assessed if accurate rates of seed-to-seedling transmission are known. Block et al. (3) ob- 
served 28 infected seedlings grown from approximately 19,735 infected seed (i.e., $0.14 \%$ seed-to-seedling transmission) when seed were produced on seed-parent plants that were inoculated with E. stewartii. When seed were produced on naturally infected seed-parent plants, one seedling was infected from approximately 4,563 infected seed (i.e., $0.022 \%$ seed-to-seedling transmission). The objective of this study was to generate additional estimates of rates of seed-to-seedling transmission of E. stewartii in order to more accurately assess the risk of introducing E. stewartii in maize seed.

\section{MATERIALS AND METHODS}

Production of infected seed. A Stewart's wilt-susceptible sweet corn hybrid, Jubilee, was selected for these experiments because symptoms of Stewart's wilt are distinct and severe on this hybrid, especially at seedling stages. Also, systemic infection of Jubilee occurs rapidly and frequently, which increases the probability that seed is infected when plants are inoculated with E. stewartii (14).

Seed infected with E. stewartii was produced on Jubilee. Pollination was not controlled. Therefore, the $F_{2}$ seedlings assayed in these trials were grown from seed produced on open-pollinated $F_{1}$ hybrids. About 0.02 ha of Jubilee was planted at the University of Illinois South Farms, Urbana on 16 May 1998, 17 May 1999, and 19 May 2000. Standard production practices were followed.

Inocula of E. stewartii were produced from a mixture of naturally occurring isolates collected in the spring and from isolates collected the previous fall that were maintained as a bulked population on infected plants in a greenhouse. Symptomatic leaves were sterilized in $95 \%$ ethanol for $10 \mathrm{~s}$ and rinsed twice in deionized water. Approximately $201.5-\mathrm{cm}^{2}$ pieces of leaf tissue were cut from symptomatic areas and placed in 1.5 liters of nutrient broth. Inocula were incubated on benchtop shakers at room temperature overnight. Nutrient broth cultures were diluted 1:10 with a $0.1 \mathrm{M} \mathrm{NaCl}$ solution prior to inoculating seedlings. Seedlings were inoculated 11, 16, 23, and 29 June 1998 and 11, 17, and 25 June 1999 using the pinprick method (5). Seedlings were not inoculated in 2000. In all 3 years, $3 \mathrm{ml}$ of undiluted $E$. stewartii inocula were injected into ear shanks of the primary ears of each plant using a modified hog vaccinator. Ear shanks were inoculated four or five times at 2- to 3-day intervals beginning 2 weeks after pollination.

Ears were harvested approximately 40 days after pollination and dried at ambient air temperatures to about $15 \%$ moisture. Seed were shelled from ears and bulked. Approximately 25, 30, and $40 \mathrm{~kg}$ of seed were produced in 1998, 1999, and 2000, respectively.
Seed were assayed for E. stewartii with a commercially available ELISA reagent set (Agdia, Inc., Elkhart, IN) that is sensitive to about $10^{5} \mathrm{CFU} / \mathrm{ml}$ (2). Seed produced in 1998 was assayed about 3 months after harvest. Seed produced in 1999 was assayed about 6 weeks before planting in 2000 . For seed produced in 2000, samples were assayed about 3 months after harvest and about 2 months prior to planting. Samples of 276, 644, and 736 kernels were selected randomly from each seed lot in 1998, 1999, and 2000, respectively. Each kernel was assayed individually.

ELISA plates were coated with antibody and incubated overnight at $4^{\circ} \mathrm{C}$. Kernels were crushed and suspended in $2 \mathrm{ml}$ of phosphate-buffered saline with Tween-20 (PBST) for $1 \mathrm{~h}$ in a small test tube. Prior to loading samples, the coating solution was hand washed from the ELISA plates four times with PBST. A 100- $\mu$ aliquot was taken from each sample, pipetted into a single ELISA plate well, and incubated in a humid box at room temperature for 2 to 2.5 h. Two positive and two negative controls were included with each ELISA plate. Positive control samples were either a 25kernel sample from seed lots known to be E. stewartii infected or plant sap extracted from infected leaf tissue. Negative controls were PBST. Plates were washed four times with PBST. The enzyme conjugate solution was added, and plates were incubated for 2 $\mathrm{h}$ in a humid box at room temperature. The enzyme conjugate solution was washed four times with PBST, and the peroxidase substrate solution was added to each well. Positive-control sample wells became distinctly colored in about 15 to $20 \mathrm{~min}$. Absorbance was measured with a Dynatech MR4000 plate reader at $490 \mathrm{~nm}$. The proportion of kernels infected with $E$. stewartii and a 95\% confidence interval were calculated from samples of 276, 644, and 736 individual kernels in 1998, 1999, and 2000, respectively, using the standard normal curve to approximate the binomial distribution.

Seedling assay for Stewart's wilt. Infected seed produced the previous year were planted 10 May 1999 in DeKalb, IL and 20 May 2000 and 18 May 2001 in Plover, WI. These locations were chosen in an attempt to prevent naturally occurring Stewart's wilt. Corn flea beetles usually are unable to survive the winter at these northern locations where the average winter temperature is below $-2^{\circ} \mathrm{C}$. Strips of sweet corn hybrids susceptible to Stewart's wilt (Supersweet Jubilee, How Sweet It Is, and Royal Sweet in 1999 and Jubilee in 2000 and 2001) were planted throughout the trials to monitor for naturally occurring Stewart's wilt. Seed of the check hybrids were produced commercially in Idaho where Stewart's wilt does not occur. In DeKalb, check hybrids were interspersed throughout the field in 12 four-row plots that were approximately $50 \mathrm{~m}$ in length. In
Plover, 1 of every 12 rows (2000) or 1 in every 6 rows (2001) in the field was planted with the check hybrid, Jubilee. Plant stands were estimated by counting the number of plants in $356.5-\mathrm{m}$ samples of rows in 1999 and 100 8-m samples of rows in 2000 and 2001. Populations of plants grown from E. stewartii-infected Jubilee seed and populations of plants grown from $E$. stewartii-free seed of check hybrids were estimated from average stands. The trial at DeKalb occupied about 0.7 ha with approximately 71,800 plants. The trials at Plover occupied about 3.0 ha with approximately 95,600 plants and 89,400 plants in 2000 and 2001, respectively.

Seedlings at the two- to three-leaf stage were examined for symptoms of Stewart's wilt 6 June 1999, 22 June 2000, and 28 June 2001. Leaf tissue was sampled from any seedling with symptoms that remotely resembled Stewart's wilt. Leaves from each sampled seedling were examined microscopically for bacterial ooze (bacteria streaming from xylem of cut leaves). All samples positive for ooze were assayed with ELISA to confirm the presence of $E$. stewartii. About 30 samples that did not ooze also were assayed with ELISA in 1999 and in 2000 as checks.

Rates of transmitting E. stewartii from seeds to seedlings were estimated by dividing the number of infected seedlings (i.e., samples that oozed and were positive from ELISA) grown from E. stewartii-infected seed by the total number of seed that were infected with E. stewartii as estimated from the ELISA tests of seed lots. Incidence of natural Stewart's wilt was estimated from the commercial check hybrids by dividing the number of seedlings positive for E. stewartii by the total number of check seedlings in the trial.

\section{RESULTS}

Infected seed. Based on ELISA tests, there were $15.6 \pm 4.3,49.4 \pm 3.9$, and 12.5 $\pm 2.4 \%$ E. stewartii-infected kernels in seed produced in 1998, 1999, and 2000, respectively (Table 1). For seed produced in 1998, 43 of 276 kernels assayed with ELISA were positive for E. stewartii. For seed produced in 1999, 318 of 644 kernels assayed with ELISA were positive for $E$. stewartii. For seed produced in 2000, 92 of 736 kernels assayed with ELISA were positive for E. stewartii.

Infected seedlings. In 1999, approximately 61,800 seedlings were grown from seed produced in 1998 on E. stewartiiinoculated plants. Approximately 10,000 seedlings of check hybrids were grown from commercial seed free of E. stewartii. Symptoms that resembled Stewart's wilt were observed on 115 of the 61,800 seedlings grown from the E. stewartii-infected seed lot. Symptomatic leaf tissue was collected from each of these seedlings. Of these 115 leaf tissue samples, 41 were 
positive for E. stewartii based on ooze tests followed by a positive ELISA test. Leaf tissue also was collected from 40 symptomatic seedlings grown from check hybrids. Of these 40 leaf tissue samples, 6 were positive for E. stewartii based on ooze tests followed by a positive ELISA test. Symptomatic leaf tissue that did not ooze was negative for $E$. stewartii when tested by ELISA.

In 2000, approximately 83,400 seedlings were grown from seed produced in 1999 on E. stewartii-inoculated plants and approximately 12,200 seedlings of the check hybrid were grown from commercial seed free of E. stewartii. Symptoms that resembled Stewart's wilt were observed on 165 of the 83,400 seedlings grown from the E. stewartii-infected seed. Of the 165 leaf tissue samples collected from these seedlings, 18 were positive for E. stewartii based on ooze tests followed by positive ELISA tests. Leaf tissue was collected from 28 symptomatic seedlings grown from the check hybrid. Bacteria did not ooze from any of these 28 leaf tissue samples and none were positive when tested by ELISA. Other symptomatic leaf tissue that did not ooze also was negative for $E$. stewartii when tested by ELISA.

In 2001, approximately 60,000 seedlings were grown from seed produced in 2000 on E. stewartii-inoculated plants and approximately 29,400 seedlings of the check hybrid grown from commercial seed free of E. stewartii. Symptoms that resembled Stewart's wilt were observed on 36 of the 60,000 seedlings grown from the $E$. stewartii-infected seed lot. Symptomatic leaf tissue was collected from each of these seedlings. None of these 36 samples oozed or were positive for E. stewartii when tested by ELISA. Leaf tissue samples were not collected from the check hybrid because Stewart's wilt symptoms were not observed.

Rates of seed-to-seedling transmission. In 1999 , about $9,600 \pm 2,650$ of 61,800 seedlings were grown from $E$. stewartii-infected seed based on an estimate of $15.6 \pm 4.3 \%$ infected kernels.
Forty-one seedlings were infected with $E$. stewartii based on symptoms of Stewart's wilt and ELISA-positive leaf tissue samples. This corresponds to a seed-to-seedling transmission rate of $0.43 \%$ (41 infected seedlings grown from 9,600 infected kernels). Based on the $95 \%$ confidence interval for the number of infected kernels from this seed lot, the rate of seed-toseedling transmission was estimated to be between 0.33 and $0.59 \%$. However, in 1999, incidence of naturally-occurring Stewart's wilt in seedlings of commercial check hybrids was about $0.06 \%$ (6 positive samples from 10,000 seedlings). If $0.06 \%$ of the 61,800 seedlings grown in 1999 from the E. stewartii-infected seed lot also were infected naturally, 37 of the $41 E$. stewartii-infected seedlings were the result of natural infection. In this case, the best estimate of the rate of seed-to-seedling transmission was $0.042 \%$ (four infected seedlings grown from 9,600 E. stewartiiinfected seed). Based on the $95 \%$ confidence interval for the number of infected kernels, the rate of seed-to-seedling transmission was estimated to be between 0.033 and $0.058 \%$.

In 2000 , about $41,200 \pm 3,250$ of 83,400 seedlings were grown from E. stewartiiinfected seed based on an estimate of 49.4 $\pm 3.9 \%$ infected kernels. Eighteen seedlings were infected with $E$. stewartii based on symptoms of Stewart's wilt and positive leaf tissue samples. This corresponds to a seed-to-seedling transmission rate of $0.044 \%$ (18 infected seedlings grown from 41,200 infected kernels). Based on the $95 \%$ confidence interval calculated for the number of infected kernels in this seed lot, the rate of seed-to-seedling transmission was estimated to be between 0.041 and $0.047 \%$.

In 2001, about 7,500 $\pm 1,320$ of 60,000 seedlings were grown from E. stewartiiinfected kernels based on an estimate of $12.5 \pm 2.4 \%$ infected kernels. None of the seedlings were positive for $E$. stewartii and seed-to-seedling transmission of E. stewartii was not observed.
When data were combined from all three trials of this experiment, about 58,300 \pm 7,220 of 205,500 seedlings were grown from E. stewartii-infected kernels. Fiftynine seedlings were infected with $E$. stewartii based on symptoms of Stewart's wilt and positive leaf tissue samples. Of these, 22 probably were due to seed-toseedling transmission of E. stewartii and 37 may have been the result of natural infection due to the presence of flea beetles in DeKalb, IL in 1999. The 22 infected seedlings from 58,300 infected kernels corresponds to a seed-to-seedling transmission rate of $0.038 \%$. Based on the $95 \%$ confidence interval calculated for the number of infected kernels, the rate of seed-toseedling transmission was estimated to be between 0.034 and $0.043 \%$.

\section{DISCUSSION}

Rates of seed-to-seedling transmission of E. stewartii observed in this study are substantially lower than seed transmission rates reported in the first half of the twentieth century $(1,10,16,17,19,21)$; however, they are similar to seed-to-seedling transmission rates reported by Block et al. (3). Our estimate of the rate of transmitting $E$. stewartii to seedlings from infected seed produced on inoculated seed-parent plants was $0.038 \%$ ( 22 of 58,300 ). Block et al. (3) estimated that $0.14 \%$ of seedlings ( 28 of 19,735) grown from E. stewartii-infected seed were infected with Stewart's wilt when seed was produced on seed-parent plants that were inoculated with $E$. stewartii. If seed was produced on seedparent plants that were infected naturally, Block et al. (3) observed a single infected seedling from 4,563 infected kernels or a seed-to-seedling transmission rate of $0.022 \%$. Thus, our estimates corroborated those of Block et al. based on a sample that was about twice as large as those from which previous estimates were calculated. Since results from these studies concur, the best estimate of seed-to-seedling transmission of E. stewartii can be calculated from data from both studies. A total of 51 in-

Table 1. Data from which rates of seed-to-seedling transmission of Erwinia stewartii were estimated, including percentage of $E$. stewartii-infected kernels, total number of seedlings emerged, estimated number of seedlings grown from E. stewartii-infected kernels, number of E. stewartii-infected seedlings, mean percentage of seedlings infected from E. stewartii-infected seed, and upper limits for the $95 \%$ confidence interval for percentage of seedlings infected from E. stewartii-infected seed

\begin{tabular}{|c|c|c|c|c|}
\hline \multirow[b]{2}{*}{ Data collected and calculated } & \multicolumn{3}{|c|}{ Year E. stewartii-infected seed planted } & \multirow[b]{2}{*}{ Total } \\
\hline & 1999 & 2000 & 2001 & \\
\hline Infected kernels $(\%)^{\mathrm{a}}$ & $15.6 \pm 4.3 \%$ & $49.4 \pm 3.9 \%$ & $12.5 \pm 2.4 \%$ & $\ldots$ \\
\hline Total seedlings emerged & 61,800 & 83,400 & 60,000 & 205,200 \\
\hline Seedlings emerged from infected seed & $9,600 \pm 2,650$ & $41,200 \pm 3,250$ & $7,500 \pm 1,320$ & $58,300 \pm 7,220$ \\
\hline Number of infected seedlings & 4 & 18 & 0 & 22 \\
\hline \multicolumn{5}{|l|}{ Infected seedlings from infected seed $(\%)^{\mathrm{b}}$} \\
\hline Mean & $0.042 \%$ & $0.044 \%$ & 0 & $0.038 \%$ \\
\hline Lower limit of $95 \%$ confidence interval & $0.033 \%$ & $0.040 \%$ & 0 & $0.034 \%$ \\
\hline Upper limit of $95 \%$ confidence interval & $0.058 \%$ & $0.047 \%$ & 0 & $0.043 \%$ \\
\hline
\end{tabular}

a Percentage of E. stewartii-infected kernels based on enzyme-linked immunosorbent assay test of 276, 644, and 736 single kernels in each of the three trials, and 95\% confidence intervals calculated from normal approximations of binomial distributions.

b Percentage of infected seedlings grown from infected seed (i.e., rate of seed-to-seedling transmission of E. stewartii). Upper and lower 95\% confidence intervals based on confidence intervals for estimates of percentage infected seed. 
fected seedlings were observed from an estimated 82,595 infected seed, which corresponds to $0.062 \%$ seed-to-seedling transmission of E. stewartii.

Our estimated rate of seed-to-seedling transmission of E. stewartii may be higher than rates that occur naturally because $E$. stewartii-infected seed used were produced by inoculating seed-parent plants. Also, the hybrid grown in our study was highly susceptible to Stewart's wilt. Block et al. (3) observed different transmission rates from infected kernels produced on inoculated plants and naturally infected plants, possibly due to an increased number of bacteria in each kernel when seed-parent plants were inoculated (3). Similarly, infected kernels in our study probably harbored a higher number of bacteria than most naturally infected seed as a result of our method of inoculating ear shanks. In recent research, absorbance values from ELISA tests were slightly higher for E. stewartiiinfected seed produced by ear shank inoculation than for seed infected naturally (C. C. Block, J. K. Pataky, and P. M. Michener, unpublished). The effect of the size of E. stewartii populations in infected kernels on seed transmission should be examined more critically; however, it is unlikely that seed infected naturally would harbor larger populations than those used in this study.

Seed-to-seedling transmission rates estimated from this study also may be higher than normal because Jubilee is extremely susceptible to Stewart's wilt. Host resistance may affect rates of seed-to-seedling transmission of E. stewartii in a manner similar to its effect on rates of plant-toseed transmission. Rates of plant-to-seed transmission (i.e., kernel infection) were affected greatly by the Stewart's wilt reaction of seed-parent plants $(4,11,14)$. Kernel infection ranged from about 2 to $70 \%$ for seed produced on highly susceptible plants that were inoculated and from about 0.8 to $35 \%$ for seed produced on highly susceptible plants that were infected naturally $(4,11,14)$. Khan et al. (11) did not detect $E$. stewartii from seed of hybrids or inbreds that were infected nonsystemically. Block et al (4) detected E. stewartii-infected seed only from plants with greater than $25 \%$ disease severity (i.e., mild systemic infection). Seed lots with greater than $5 \%$ kernel infection came from plants with greater than $50 \%$ disease severity (i.e., severe systemic infection; 4). Michener et al. (14) detected about $0.1 \%$ kernel infection from inoculated plants with moderate reactions to Stewart's wilt and about $0.01 \%$ kernel infection from inoculated plants with resistant reactions. If rates of seed-to-seedling transmission are affected by host resistance in a manner similar to rates of plant-toseed transmission, our estimates and those of Block et al. (3) are among the highest that are likely to occur because of the extreme susceptibility of the plants used in these studies.
Failure to observe infected seedlings in the grow-out trial in Plover, WI in 2001 did not substantially alter our conclusions. If rates of seed-to-seedling transmission were near $0.04 \%$ and 7,500 of the 60,000 seedlings that emerged in this trial were grown from infected seed, we would have expected to find on average only 3 infected seedlings in the trial. Possibly, an infected seedling or two may have been missed during the 3 days that two people scouted this 3-ha field. Alternatively, it is possible that none of the seedlings were infected. Based on the binomial distribution for $p=$ $0.00038, q=0.99962$, and $n=7500$, there is about a $6 \%$ chance (i.e., $q^{n}=$ $0.99962^{7,500}$ ) that none of the seedlings were infected if our assumptions about kernel infection and seed-to-seedling transmission were correct.

The absence of infected seedlings in the trial in 2001 also may have been due to lower rates of seed-to-seedling transmission when incidence of infected kernels is low. Block et al. (3) observed only a single instance of seed-to-seedling transmission of E. stewartii from seed lots with less than $35 \%$ infected kernels. In our study, seed-toseedling transmission was not observed in 2001 when incidence of infected seed was $12.5 \%$. In 2000 , when $45 \%$ of the seed were infected, the rate of seed to seedling transmission was $0.044 \%$. In 1999 , results were confounded by natural infection. We assumed that $0.06 \%$ of the seedlings ( 37 of 61,800 plants) grown in 1999 from the $E$. stewartii-infected seed lot were infected by flea beetles which was the same rate of natural infection we observed in check plants grown from commercial seed free of E. stewartii. If $0.066 \%$ of the plants in this field were naturally infected rather than $0.06 \%$, none of the Stewart's wilt-infected plants were the result of seed transmission. The $95 \%$ confidence interval for our $0.06 \%$ estimate of naturally infected plants was $\pm 0.048 \%$. Therefore, it would not be improbable to conclude that we did not observe seed-to-seedling transmission in 1999 or 2001 when incidence of kernel infection was 15 and $12.5 \%$, respectively. Additional research on the association between levels of kernel infection and rates of seed-to-seedling transmission may be useful in defining thresholds of kernel infection for quarantine purposes.

In our trials, many seedlings with symptoms that remotely resembled Stewart's wilt were not infected by E. stewartii. Of 356 symptomatic leaf samples collected, bacterial ooze was observed microscopically from 65 samples. Observations of ooze were correlated perfectly with positive tests for E. stewartii using a commercial ELISA kit. All 65 samples that oozed had positive ELISA tests. The ELISA test was negative for 124 samples that did not ooze. Block et al. (3) also observed wilted seedlings from which E. stewartii could not be detected. They suggested that seed- ling wilt symptoms caused by ear rot fungi such as Diplodia zeae, Penicillium oxalicum, Fusarium moniliforme, and Gibberella zeae may have contributed to the unusually high estimates of E. stewartii-seed transmission in the first half of the twentieth century. Our observations support this hypothesis.

Rates of plant-to-seed transmission and rates of seed-to-seedling transmission (13) affect seed transmission (13). Different rates of seed transmission of E. stewartii would be expected for seed produced on resistant, moderate, and susceptible seedparent plants based on our estimates of variable rates of plant-to-seed transmission (14) and estimates of seed-to-seedling transmission of about $0.06 \%$ based on the combined data of this study and those of Block et al. (3). If seed infection is $10 \%$ when seed is produced on susceptible seedparent plants for which incidence of Stewart's wilt infection is $100 \%$, we would expect to observe 1 infected seedling in about 16,700 based on $0.06 \%$ seed-toseedling transmission. If seed infection is $0.1 \%$ when seed is produced on seed-parent plants with moderate reactions to Stewart's wilt and incidence of Stewart's wilt is $100 \%, 1$ of about 1,666,700 seedlings may be infected. For resistant hybrids, one seedling may be infected in about $16,666,700$ if $0.01 \%$ of seed are infected when seed is produced in fields in which $100 \%$ of the seed-parent plants are infected with E. stewartii.

Considering the levels of Stewart's wilt resistance in most corn hybrids and inbreds grown presently in the United States, the risk of introducing E. stewartii to areas where it is not endemic through seed or grain is exceedingly low. Phytosanitary regulations that restrict the movement of maize seed and grain should consider the results of recent studies on rates of seed transmission of E. stewartii rather than relying on the $2 \%$ seed transmission rate that has been repeated in the literature but appears to be inaccurate for seed and grain of modern corn hybrids.

\section{ACKNOWLEDGMENTS}

We thank L. Paul (UIUC Northern Illinois Agronomy Center) for assistance with grow-out trials in DeKalb, IL and J. Wegner (Illinois Foundation Seeds, Inc.) for assistance with growout trials in Plover, WI.

\section{LITERATURE CITED}

1. Blakemore, E. J. A., Law, J. R., and Reeves, J. C. 1999. PCR identification of Erwinia stewartii and its comparison with two other methods. Seed Sci. Technol. 27:385-396.

2. Blakemore, E. J. A., Reeves, J. C., and Ball, S. F. L. 1992. Polymerase chain reaction used in the development of a DNA probe to identify Erwinia stewartii, a bacterial pathogen of maize. Seed Sci. Technol. 20:331-335.

3. Block, C. C., Hill, J. H., and McGee, D. C. 1998. Seed transmission of Pantoea stewartii in field and sweet corn. Plant Dis. 82:775780 . 
4. Block, C. C., Hill, J. H., and McGee, D. C. 1999. Relationship between late-season severity of Stewart's bacterial wilt and seed infection in maize. Plant Dis. 83:527-530.

5. Chang, C. M., Hooker, A. L., and Lim, S. M. 1977. An inoculation technique for determining Stewart's bacterial leaf blight reaction in corn. Plant Dis. Rep. 61:1077-1079

6. Coplin, D. L., Majerczak, R., Zhang, Y., Kim, W.-S., Jock, S., and Geider, K. 2002. Identification of Pantoea stewartii subsp. stewartii by PCR and strain differentiation by PFGE. Plant Dis. 86:304-311.

7. Elliott, C. 1941. Bacterial wilt of corn. In: USDA Farmers' Bull. 1878.

8. Elliott, C., and Poos, F. W. 1934. Overwintering of Aplanobacter stewartii. Science 80:289-290.

9. Elliott, C., and Poos, F. W. 1940. Seasonal development, insect vectors, and host range of bacterial wilt of sweet corn. J. Agric. Res.
60:645-686.

10. Ivanhoff, S. S. 1933. Stewart's wilt disease of corn, with emphasis on the life history of Phytomonas stewartii in relation to pathogenesis. J. Agric. Res. 47:749-770.

11. Khan, A., Ries, S. M., and Pataky, J. K. 1996. Transmission of Erwinia stewartii through seed of resistant and susceptible field and sweet corn. Plant Dis. 80:398-403.

12. Lamka, G. L., Hill, J. D., McGee, D. C., and Braun, E. J. 1991. Development of an immunosorbent assay for seedborne Erwinia stewartii in corn seeds. Phytopathology 81:839-846.

13. McGee, D. C. 1995. Epidemiological approach to disease management through seed technology. Annu. Rev. Phytopathol. 33:445446

14. Michener, P. M., Pataky, J. K., and White, D. G. 2002. Transmission of Erwinia stewartii from plants to kernels and reactions of corn hybrids to Stewart's wilt. Plant Dis. 86:161172.

15. Pepper, E. H. 1967. Stewart's Bacterial Wilt of Corn. Monogr. No. 7. American Phytopathological Society, Worcester, MA.

16. Rand, F. V., and Cash, L. C. 1921. Stewart's disease of corn. J. Agric. Res. 21:263- 264.

17. Rand, F. V., and Cash, L. C. 1933. Bacterial wilt of corn. USDA Tech. Bull. No. 362.

18. Robert, A. L. 1955. Bacterial wilt and Stewart's leaf blight of corn. USDA Farmer's Bull. 2092.

19. Smith, E. F. 1909. Seed corn as a means of disseminating Bacterium stewartii. Science 30:223-224.

20. Stevens, N. E. 1935. Stewart's disease in relation to winter temperatures. Plant Dis. Rep. 18:141-149.

21. Thomas, R. C. 1924. Stewart's disease or bacterial wilt of sugar corn. Ohio Agric. Exp. Stn. Month. Bull. 9:81-84. 